\title{
Towards practical reactive security audit using extended static checkers
}

\author{
Julien Vanegue \\ Bloomberg L.P. \\ New York, NY, USA \\ jvanegue@bloomberg.net
}

\author{
Shuvendu K. Lahiri \\ Microsoft Research \\ Redmond, WA, USA \\ shuvendu@microsoft.com
}

\begin{abstract}
This paper describes our experience of performing reactive security audit of known security vulnerabilities in core operating system and browser COM components, using an extended static checker HAVOC-LITE. We describe the extensions made to the tool to be applicable on such large $\mathrm{C}++$ components, along with our experience of using an extended static checker in the large. We argue that the use of such checkers as a configurable static analysis in the hands of security auditors can be an effective tool for finding variations of known vulnerabilities. The effort has led to finding and fixing around 70 previously unknown security vulnerabilities in over 10 millions lines operating system and browser code.
\end{abstract}

Keywords-security audit; program verification; static analysis; extended static checkers

\section{INTRODUCTION}

Ensuring security of software has become of paramount importance to the software industry. Every software development group, representing either a small team of developers or an entire company, mandates extensive testing and analysis to safeguard against security breaches. However, security flaws will remain a part of life, at least in the case of legacy applications that cannot be redesigned from scratch. In such cases, effective defense mechanisms are required to mitigate the impact of security vulnerabilities. In particular, finding all possible variants of a known security vulnerability can go a long way in safeguarding the known attack surface of a software system.

The Microsoft Security Response Center (MSRC) identifies, monitors, resolves, and responds to security incidents and $\mathrm{Mi}$ crosoft software security vulnerabilities. The following quote summarizes some of the activities that the team performs in conjunction with product teams to mitigate future occurrences of known vulnerabilities that merit security bulletins: ${ }^{1}$

"...The MSRC engineering team investigates the surrounding code and design and searches for other variants of that threat that could affect customers."

This is an expensive and arduous process that involves a mix of manual testing, fuzzing and a large amount of manual security audit. Such audits need to be responsive and timely in order to prevent attackers from crafting similar attacks in the near future. We define the term reactive security audit as this

${ }^{1}$ http://www.microsoft.com/security/msrc/whatwedo/updatecycle.aspx process of performing effective audit for variants of known vulnerabilities, over a large code base. Although the current practices are effective, they leave a lot to be desired in terms of scalability and confidence obtained after the reviews.

In this work, we explore the use of extended static checking tools towards improving the productivity of auditors performing reactive security audit, and increasing the confidence of the audit $^{2}$. Extended static checking tools (such as ESC/Java [1], HAVOC [2]) offer a potential to develop configurable static analysis tools with high coverage guarantees. These tools provide the user the ability to write contracts (specifications of procedures) in the form of preconditions, postconditions, assertions and discharge them using modern Satisfiability Modulo Theories (SMT) solvers [3]. The semantics of the source language is precisely defined once by the tool (and does not vary by the property being checked), and the assumptions are well documented. Many such tools are also equipped with simple yet robust user-guided contract inference tools (such as Houdini [4]) to reduce the manual overhead of writing simple intermediate contracts. Unlike full functional correctness verifiers (such as VCC [5]), they make pragmatic assumptions to reduce the complexity of proofs, and provide a lot more automation in the form of inference. Although the use of extended static checkers had been proposed for ensuring security a decade back [6], not much success has been reported in practical usage. Our conjecture is that the absence of a usable, robust and scalable tool for the space of core operating system and browser implementations has been one of the main causes for the lack of adoption.

In this paper, we present a case study of using an extended static checker HAVOC-LITE ${ }^{3}$ for checking variants of security vulnerabilities in Microsoft Windows and Internet Explorer. We document the challenges encountered in deploying the previous version of the tool (henceforth called HAVOC) and the extensions needed to apply the tool in a realistic setting. The extensions include modeling most common $\mathrm{C}++$ language features used typically in such applications, scaling the contract inference to be applicable to modules with several hundred thousand procedures, and early annotation validation. We then

\footnotetext{
${ }^{2}$ The work was done when the first author was employed at Microsoft.

${ }^{3}$ HAVOC-LITE is the new version of HAVOC [2] developed to meet the needs of this deployment.
} 
present our experience of security engineers using the tool on several properties devised as a response to several existing vulnerabilities. Over the course of one year, the effort led to discovering and fixing around 70 previously unknown security vulnerabilities in over 10 million lines of production code. We discuss the effort involved in modeling, performing inference of annotations and dealing with false alarms encountered along the way. We conclude that in spite of the current limitations, such a tool can be (and already is) quite cost-effective in complementing existing efforts based on fuzzing and manual audit. We conjecture that it can improve the productivity of security auditors who invest in implementing ad-hoc tools to aid their manual audit.

The rest of the paper is organized as follows:

1) In Section II, we present an overview of our approach using two simplified examples. The examples illustrate some of the challenges posed when analyzing low-level systems code, a brief summary of the modeling effort, dealing with object-oriented features and the use of annotation inference.

2) In Section III, we provide a brief description of the existing tool HAVOC that has been applied to large Windows modules to find errors. In Section IV, we describe the main shortcomings of HAVOC that limited its application for our problem domain. We describe the design of HAVOC-LITE that includes modeling an expressive subset of $\mathrm{C}++$ features, scaling the annotation inference by using a two-level algorithm that avoids memory blowup for large modules, along with other features required for making the tool robust and usable in the hands of security auditors.

3) In Section $V$, we describe the effort of applying HAVOC-LITE on the core OS and browser components. We discuss the properties that were modeled as variants of existing security vulnerabilities, candidate annotations required for inferring intermediate annotations, and some representative errors. We show the need for various pragmatic decisions (such as dealing with unsound modifies clause) to trade off soundness for costeffectiveness of the analysis. We highlight the effort required in devising the inference to reduce false alarms and the payoff over the different properties.

4) In Section VI, we discuss other related static analysis tools, and finally conclude in Section VII.

\section{MOTIVATING EXAMPLES}

In this section, we introduce two concrete examples containing commonly found programming style in $\mathrm{C}++$ and $\mathrm{COM}$ (Component Object Model [7]) applications. These examples can only be precisely analyzed if the semantics of bit-level manipulations are properly modeled, the common objectoriented and interface-oriented programming are well handled by the extended static checker.

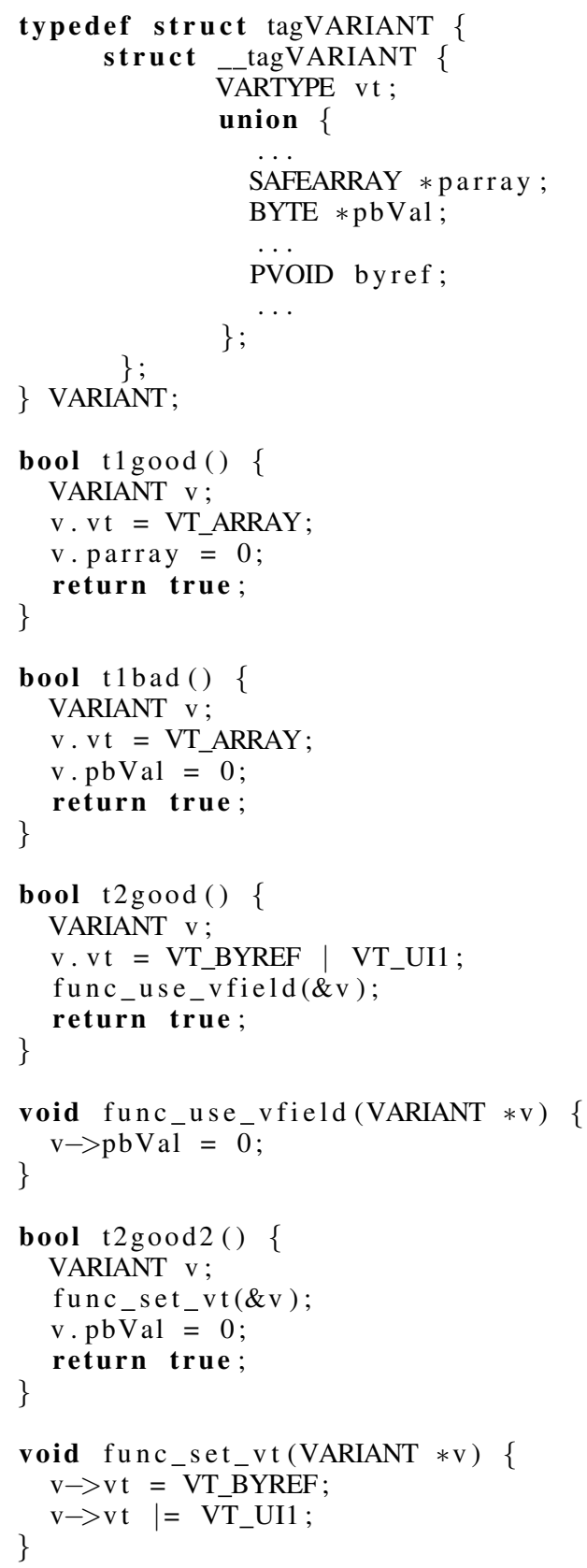

Fig. 1. Example of analysis requiring inter-procedural bit-level reasoning

\section{A. Example 1: Inter-procedural and bit-precise reasoning}

The first example shows a generic container data structure called a VARIANT and commonly used in $\mathrm{C}++$ /COM applications. This structure contains a special field $\mathrm{vt}$ and a union of data attributes. The value of the vt field indicates the union field that is correctly initialized. Failure to check the value of the vt field can lead to using the wrong union field and therefore may use a pointer field with an invalid integer value. Such mistake is likely to lead to a security vulnerability (such as in functions $t 1 \mathrm{bad})$. The check for the vt field and the 
use of the associated union field are often done in separate functions (such as in $t 2 \mathrm{good}$ and $t 2 \mathrm{good} 2$ ). Therefore, constraints must be adequately propagated inter-procedurally to avoid false positives from static analysis warnings. In addition, since the vt attribute is a bit field, bit-level program semantics needs to be supported by the static checker. Finally, a user needs to be able to document the desired property by checking each dereference of the set of fields (such as pbarray, pbVal) under consideration. Such features are supported by HAVOC-LITE and was used to find multiple security vulnerabilities in a critical browser component.

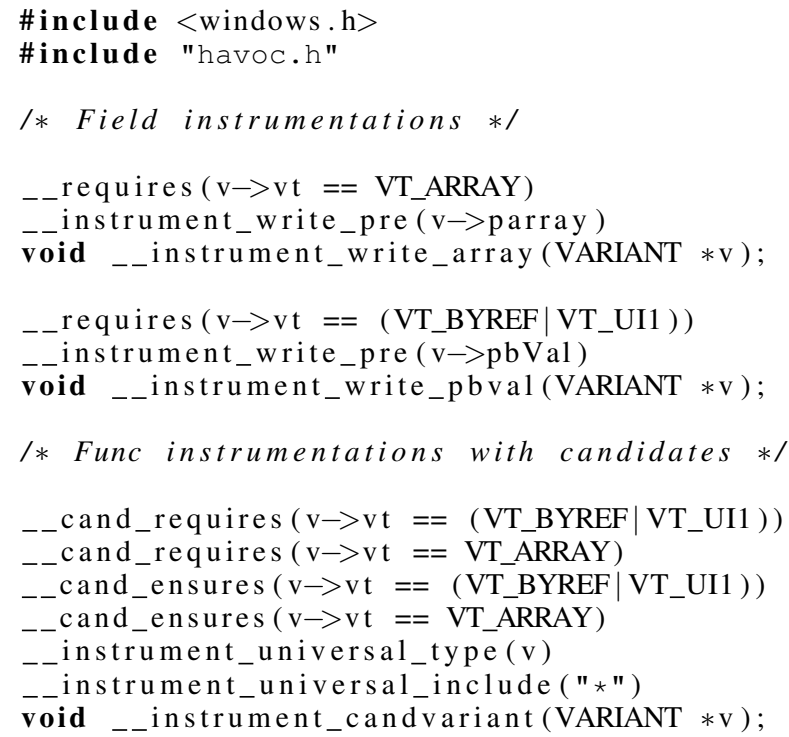

Fig. 2. Annotations for the first example.

Figure 2 shows the annotations written by the user to create a checker for this property. There are two parts to the annotations: (a) devising the property and (b) creating an interprocedural inference.

The "Field instrumentations" are instrumentations provided to insert an assertion at reads to particular fields. For example, the instrumentation provided using instrument_write_array method (the name of the method can be arbitrary except for the prefix _instrument_) specifies that every write to the field parray in the structure VARIANT is preceded by ("write_pre") an assertion that the vt field in the structure equals the value VT_ARRAY. There is a similar check before writes to the pbVal field using the instrumentation_instrument_write_pbVal. These two instrumentations allow the user to document the property to be checked.

We now look at how the user can configure the interprocedural annotation inference. The "Func instrumentations" are used to write annotations on a set of methods, instead of listing each individual method. The instrumentation primitive__instrument_universal_type specifies that any method that takes a parameter of type VARIANT * is instrumented. The filter instrument_universal_include can be used to restrict the instrumentation to only methods whose names match a particular pattern - in this case, the wildcard pattern "*” matches any method. The annotations in cand_requires and_cand_ensures are candidate preconditions and postconditions. These candidates are fed to the inter-procedural annotation inference engine that infers a subset of them as annotations that actually hold on every context.

When the user runs HAVOC-LITE with the property and candidate annotations, the tool infers two annotations: The func_use_vfield has a precondition

requires (v->Vt $==$ (VT_BYREF | VT_UI1))

and the method func_set_vt has a postcondition

_ensures (V->Vt $==$ (VT_BYREF | VT_UI1))

These additional annotations allow the tool to only complain about the method $t 1 \mathrm{bad}$, which corresponds to the only true error. Although for this simple example, it is easier to simply write the two additional annotations, it is immensely useful when dealing with modules with several thousand deeply nested procedures.

\section{B. Example 2: Object-oriented reasoning}

A second example involves the IUnknown interface class which is responsible for all the reference counting mechanisms necessary to maintain object consistency in COM applications. In this example, the class method A::Action performs a call to QueryInterface which is in charge of retrieving an instance of the interface given an input interface identifier. Such a call performs an implicit call to AddRef which increases the reference counter for this interface. Success of this call (when the return status is S_OK) leads to calling method WebAction which performs the expected operation on class B. Failure to retrieve the interface methods leads to early termination, where the IUnknown interface pointer is released using a call to method ReleaseInterface, which is in charge of decrementing the reference count for this interface (provided the interface pointer is non-NULL).

A security vulnerability exists in this example due to the lack of NULL initialization of the IWebI face pointer, which leads to corrupting the reference counter of an unknown location in the program in case the call to Query Interface is not successful. Such example requires an accurate objectoriented awareness from the static checker. We later show how HAVOC-LITE was used to uncover multiple similar security vulnerabilities in a critical browser component.

To model this property, the user can introduce a ghost field Queried in every object — the ghost field tracks whether an object has been created by a successful call to QueryInterface. The value of the ghost field for an object 


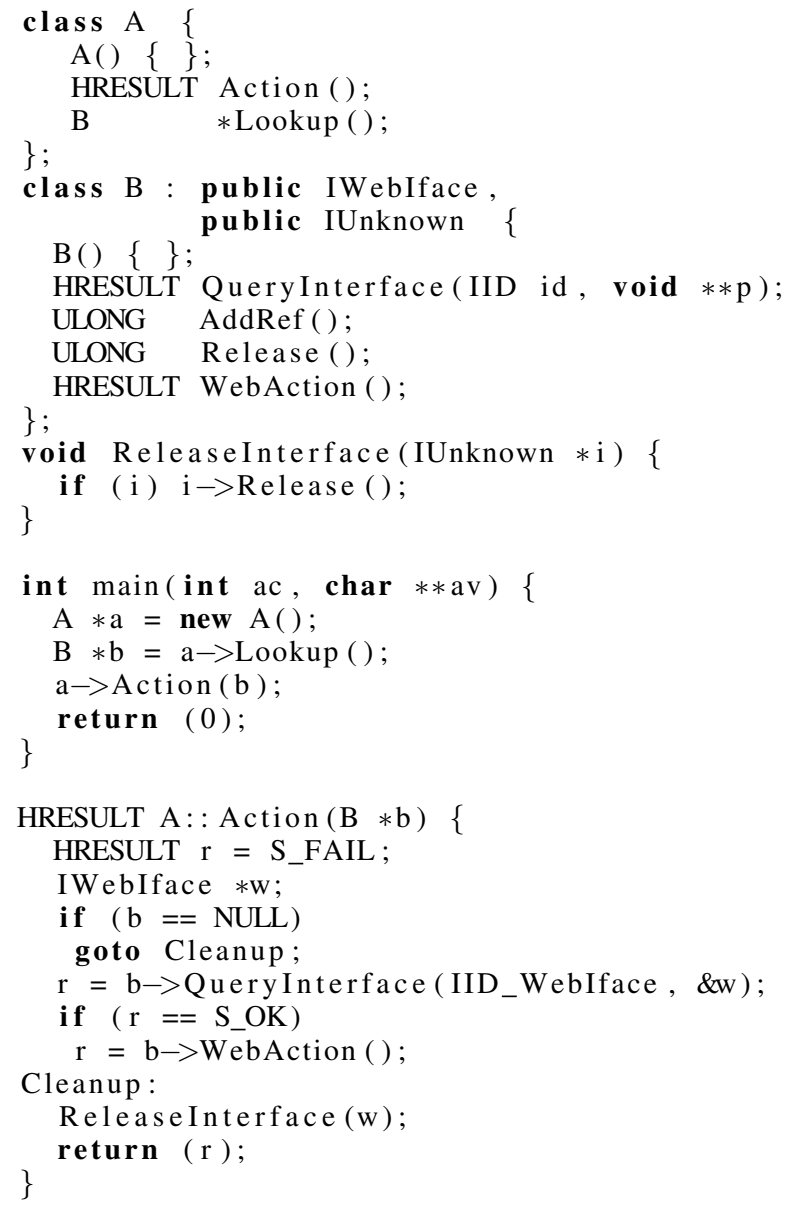

Fig. 3. Example of analysis requiring precise object-oriented semantics

$\mathrm{x}$ is written as _resource ('Queried'', $\mathrm{x}$ ). One can write a precondition for the Release methods of $B$ and any of its derived classes:

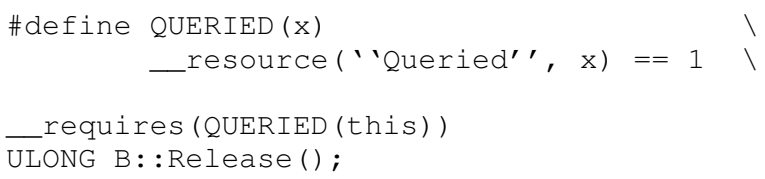

to indicate that the receiver object of the method Release has to be created by an earlier call to QueryInterface. This is in turn modeled by writing the following postcondition for the method:

Eensures (_return $\left.!=S_{\text {OK }}|| \operatorname{QUERIED}(* \mathrm{p})\right)$
HRESULT B: QueryInterace (IID id, $\operatorname{void} * * \mathrm{p})$;

where _return denotes the return value of the procedure.

Finally, one needs to infer annotations such as:

_requires $(i==$ NULL || QUERIED(i))

void ReleaseIface (IUnknown *i);

which can be done with the help of the first populating candidate annotations on all methods that consume a IUnknown as an argument (we do not show the instrumentation here), and then performing inter-procedural annotation inference.

\section{BACKGROUND: HAVOC}

In this section, we provide a background on HAVOC. We describe HAVOC-LITE along with the extensions created for this paper in Section IV.

HAVOC can be best thought of as an extended static checker for C programs, in the spirit of ESC/Java [8]. It provides an (a) extensible property checker with the aid of an annotation/contract language, (b) a procedure modular verifier that provides an accurate depiction of $\mathrm{C}$ semantics, (c) an user-guided interprocedural annotation inference engine, along with (d) various instrumentation primitives. Figure 4 shows the overall usage model of HAVOC (and also of HAVOC-LITE) in the hands of a user. We describe each of the components briefly in the next few subsections. More details about these features can be found in an earlier work [2].

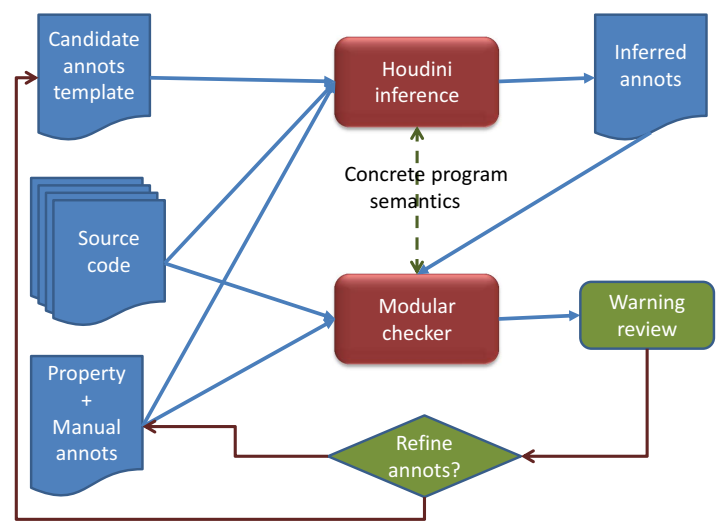

Fig. 4. HAVOC and HAVOC-LITE flow.

\section{A. Contract language}

A user can document contracts about the code using an annotation language. An annotation is an assertion over the state of the program. There are four classes of assertions (i) assertions _assert (e), (ii) assumptions _assume (e), (iii) preconditions _requires (e) and (iv) postconditions _ensures (e). Here $e$ refers to a side-effect-free $\mathrm{C}$ expression that evaluates to a scalar or pointer value - in other words, $e$ cannot be a structure value. For example, one can write _ensures $(* x==y->f)$ to indicate that on exit from a procedure the value obtained by dereferencing the variable $x$ is identical to the value stored in a field $f$ inside the structure pointed to by $y$. In addition to assertions, a user can specify a __modifies clause that specifies which part of the heap is modified by a procedure. For the purpose of 


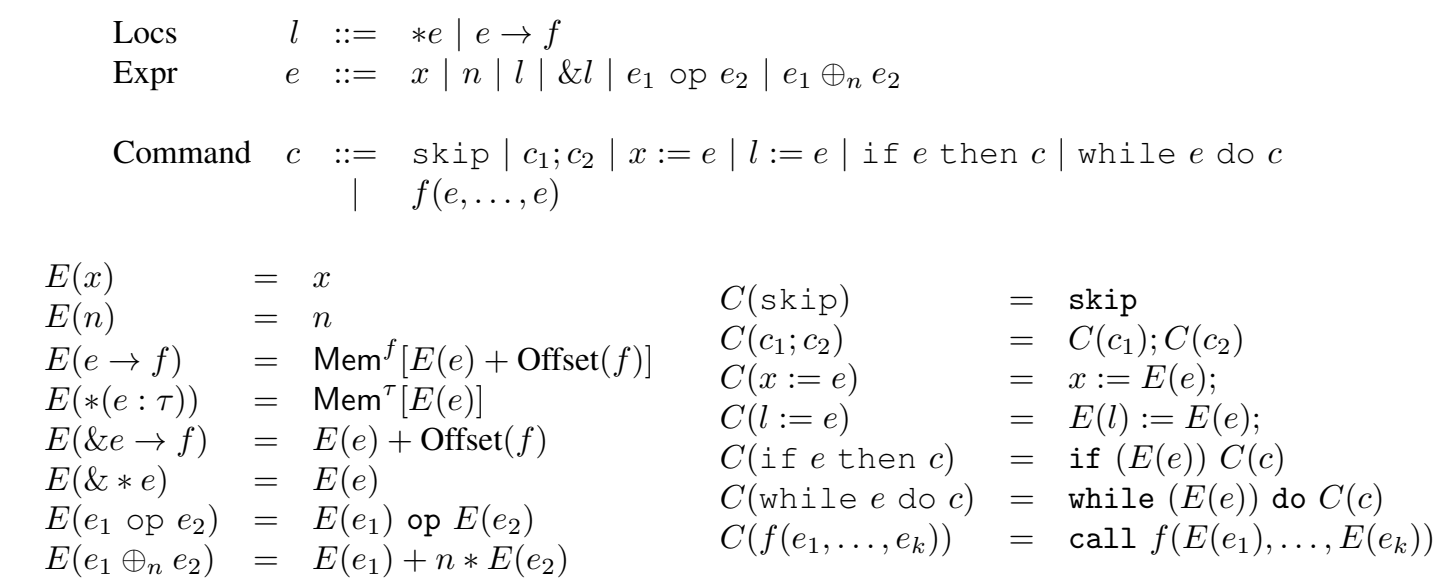

Fig. 5. A simplified subset of $\mathrm{C}$, and translation from $\mathrm{C}$ into BoogiePL. $E()$ maps a $\mathrm{C}$ expression into BoogiePL expression and $C()$ maps a $\mathrm{C}$ statement into a BoogiePL statement.

this paper, we ignore discussion on loop invariants, which are also supported in HAVOC.

In addition to the expressions in scope, a user can refer to the return variable by using the symbol _return in a postcondition, refer to the value of an expression at entry to a procedure using _old () in a postcondition. For example, _ensures (_return $==$ _old $(* x)+1)$ signifies that the value of return variable is one more than the value stored in $\star x$ at the entry to the procedure. The user can also refer to the state of ghost fields that augment the state of the program using a keyword _resource ${ }^{4}$. The scalar expression _resource $(s, e)$ where $s$ is a string and $e$ is a side-effect pointer/scalar expression, refers to the value of a ghost field named "s" inside the structure pointed to by $e$; i.e. the value of $e^{->s}$. The user can modify such ghost fields in the program.

The annotation language, along with the presence of ghost fields allows user to encode various interesting properties of the program. Section II provides a few examples of such properties. Several more examples of properties can be found in earlier works [2].

HAVOC provides a sufficiently accurate memory model for $\mathrm{C}$ programs that provides meaning to constructs such as pointer arithmetic, casts, yet supports common disambiguation required for scalable reasoning of high level properties. Figure 5 provides a simplified subset of $\mathrm{C}$ (for illustration purposes) without nested structures and addresses of variables. Figure 5 also provides the translation of this subset into an intermediate verification language BoogiePL [9]. BoogiePL is a simple procedural language, where the set of variables are restricted to Booleans, integers and arrays over them. The operator $C()$ maps a $\mathrm{C}$ statement to the equivalent BoogiePL statement in a straightforward manner. In addition, the language has support for assertions, assumptions, preconditions and postconditions - the HAVOC annotations map directly to them.

${ }^{4}$ Ghost variables are also supported as a degenerate case of ghost fields.
The operator $E()$ maps a $\mathrm{C}$ expression (present in either a statement or an annotation) into a BoogiePL expression. The heap is split into a finite number of arrays (named $\mathrm{Mem}^{f}[]$ or $\mathrm{Mem}^{\tau}[]$ ), one per scalar field or pointer field $(f)$ or pointer type $(\tau)$. Dereferencing a pointer is modeled as indexing into the appropriate array with a suitable offset - the operator Offset $(f)$ provides the offset of the field $f$ inside its parent structure. The heap splitting assumes field safety [10] that allows exploiting the types and fields in the program to get disambiguation. Under field safety, it is (optimistically) assumed that $\& x \rightarrow f$ can never alias with $\& y \rightarrow g$ for distinct field names $f$ and $g$. Further, addresses of a field $\& x \rightarrow f$ does not alias with the address $\& * e$. Although HAVOC has an option to not assume field safety, the annotation overhead increases several fold even for simple examples. In our experience, we have seldom found this optimistic assumption to limit our ability to find violations of security properties described in this paper.

\section{B. Modular verifier}

An annotated BoogiePL program is checked for correctness one procedure at a time by the Boogie program verifier. The verifier uses verification condition generation (translation of the program into a logical formula with near linear size) [11] and automated theorem proving (namely Satisfiability Modulo Theories solvers [3]) to check the satisfiability of the formula. HAVOC lifts an intraprocedural counterexample at the BoogiePL level to display over the $\mathrm{C}$ source code. In our experience, the ability of Boogie to generate a compact verification for the theorem prover is key to the scalability of HAVOC compared to tools that employ symbolic execution based path enumeration. The theorem prover can internally perform complex reasoning to prune parts of a procedure (with often several thousand paths) that are not relevant towards proving an assertion. 


\section{User-guided inference}

HAVOC uses a variant of the Houdini algorithm to perform inter-procedural inference of procedure annotations [4]. A user can write a set of candidate preconditions (_cand_requires (e)) and postconditions (__cand_ensures (e)) in addition to the usual annotations. The Houdini algorithm performs an inter-procedural greatest fix-point analysis to retain the (unique) maximum subset of these candidates that can be proved modularly by the program verifier, while assuming the non-candidate annotations. The fix-point proceeds by maintaining a worklist of procedures to be checked. At each step, a procedure $p$ is checked using the modular verifier. Any candidate annotation that cannot be proved is removed from the list of annotations. Depending on the nature of the removed candidate, either the callers of $p$ (for candidate postcondtiions), or the callee $q$ (whose candidate preconditions are removed) are added to the worklist, in addition to $p$. The process is repeated until the worklist is empty. The simple algorithm terminates in at most $n * c$ iterations, where $n$ is the total number of procedures in the module, and $c$ is the total number of candidate annotations.In practice, it runs almost linear in $c$, thus guaranteeing a quick turnaround.

\section{Instrumentations}

Finally, various syntax-based instrumentation facilities are provided to avoid manually writing annotations on large codebases. For example, an user can instrument all procedures whose names match a regular expression with a postcondition on globals, or instrument all procedures that take a parameter $x$ of type $\tau *$ by a precondition parameterized by $x$. Moreover, these annotations can include candidate annotations as well - this is crucial to performing the annotation inference. In addition to procedure annotations, the user can also instrument reads and writes to specific fields, types or globals to insert an assertion or assumption before or after the source line (as illustrated in Section II).

\section{HAVOC-LITE}

Although HAVOC had earlier been applied to large Windows source modules [2], we found it lacking in terms of usability and applicability for the code bases under investigation. The principal limitations that we addressed and describe in this section are: (i) need for modeling common $\mathrm{C}++$ language constructs, (ii) augmenting the instrumentation mechanisms to exploit $\mathrm{C}++$ class structure, (iii) scaling the annotation inference to modules containing hundreds of thousands of procedures, and several other usability concerns such as the need to modify source code to insert annotations. In the process of making the tool more robust, we dropped support for some of the earlier features in HAVOC (hence the name HAVOC-LITE) such as checking type-safety of C programs, using fine-grained modifies clauses, dealing with linked list invariants and checking for complex modifies clauses in candidate annotations described in earlier works [12].

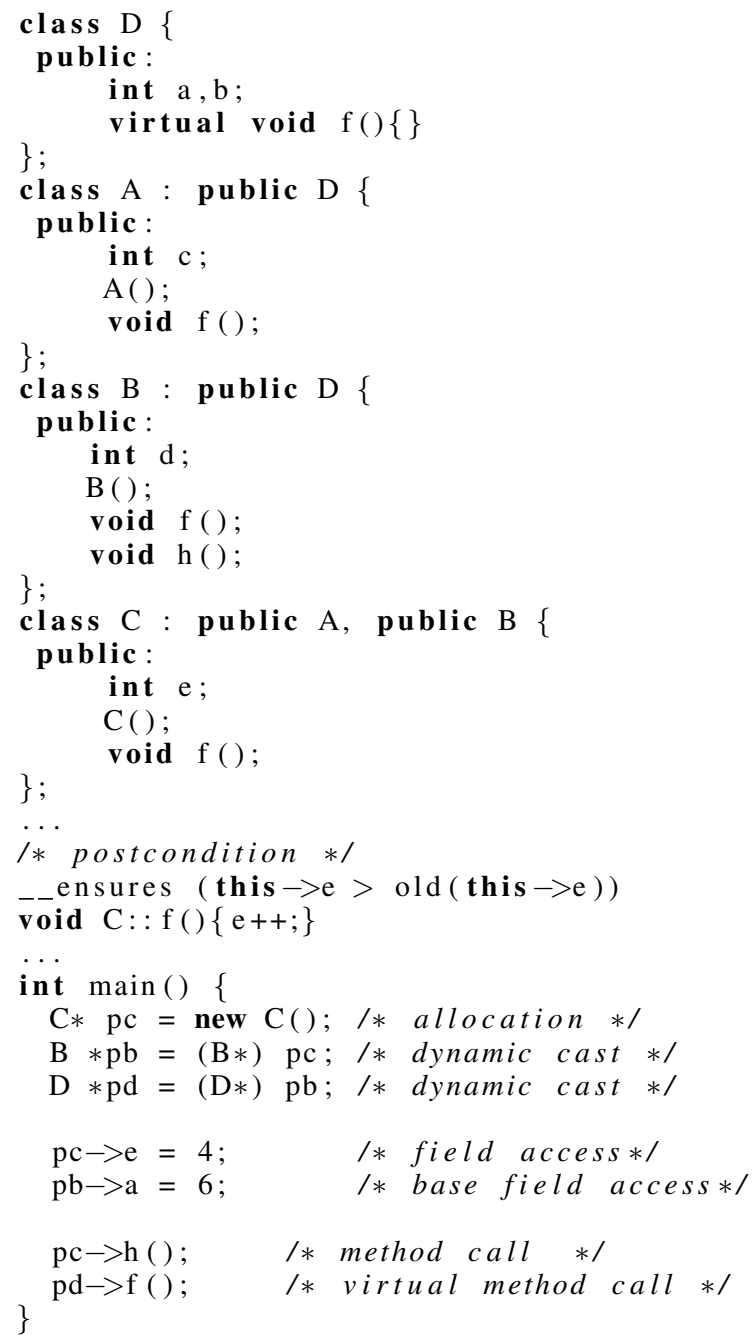

Fig. 6. A simple $\mathrm{C}++$ example.

\section{A. Modeling $C++$ language constructs}

By far, the most significant shortcoming of HAVOC was inability to deal with most $\mathrm{C}++$ constructs. In this section, we briefly describe some of the changes required to handle the most common $\mathrm{C}++$ features used commonly in the COM components. We illustrate the translation of $\mathrm{C}++$ to BoogiePL program with the aid of a simple example in Figure 6 and the translated BoogiePL program in Figure 7.

To handle instance methods, we make the receiver object explicit by exposing the this pointer. The BoogiePL translation of the procedure $C:: f$ takes this as an argument, and updates the field array Mem_e that represents the field $e$ in the class. We use the function Offset_f_C to denote the offset of a field $f$ in a class C. Further, the annotation expressions can refer to this in assertions, as shown for this method.

Constructor calls are modeled by first allocating a contiguous buffer with the size of the class by invoking the special method__HV_malloc. The mutable variable alloc 


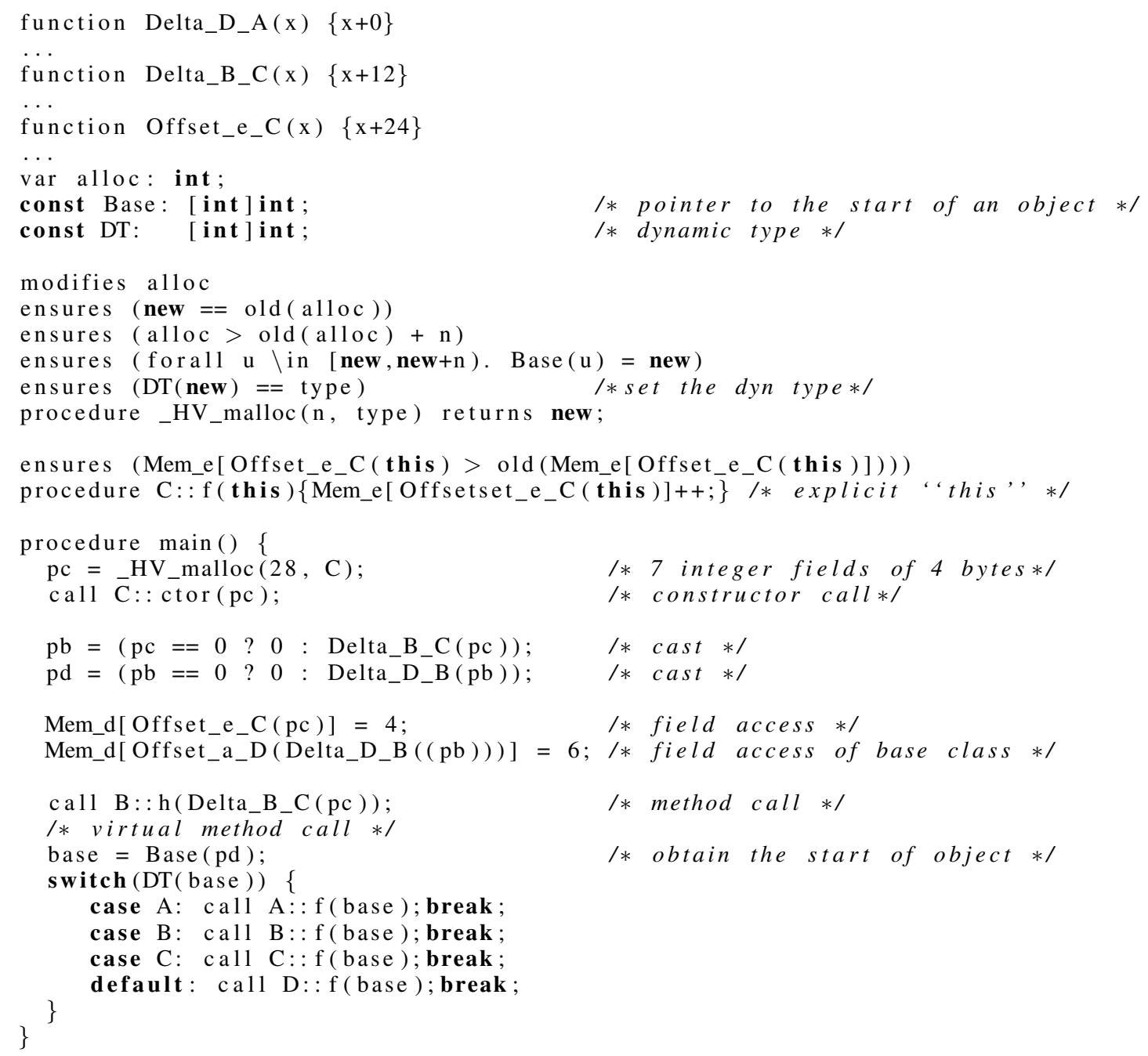

Fig. 7. The BoogiePL (cleaned up) for the $\mathrm{C}++$ example.

monotonically increases to ensure that the buffer allocated is fresh. $^{5}$ The constant map Base tracks the base of the buffer for any pointer in the buffer, and the constant map DT maps the base of the buffer to its dynamic type (the type used during allocation). The constraints are enforced by the specification of the procedure __HV_malloc specified using the _ensures annotations. The newly allocated object is then passed to the constructor of the class.

Dynamic casts is modeled by a conditional assignment that checks if the right hand side (RHS) of the assignment is null or not. In case, the RHS is non-null, it assigns the pointer shifted by the offset of the base class in the derived type. The functions Delta_A_B model the offset of a base class A inside a derived class $B$.

Field access is modeled similar to $\mathrm{C}$ programs as before, except for accessing fields inside base classes. To access fields in a (transitive) base class (such as the field a defined within the base class $D$ from within $B$ ), we first add the offset of

${ }^{5}$ We currently do not model deallocation. the base classes in the access path from the derived class (Delta_D_B for this example).

Non-virtual method calls are handled similar to C, except the addition of the this parameter. Similar to field access, calling a method in one of (transitive) base classes requires the addition of the offsets of the base classes in the access path. Virtual method calls require looking up the dynamic type (stored in DT) of the start of the object into a temporary variable base and performing a case split on the possible set of runtime types. For each dynamic type, the corresponding virtual method is invoked (assuming for simplicity the method is defined in each derived class). In each of the cases, the pointer passed as the this parameter is the start of the object stored in the map Base during allocation. Currently, we assume a separate analysis to compute the set of potential target types that is fed into HAVOC-LITE as an input.

In addition, HAVOC-LITE also handles operator overloading, simple forms of templates (both parameterized by types or values) and other $\mathrm{C}++$ features commonly encountered in 
COM. Although the modeling is far from being considered complete for $\mathrm{C}++$, it allows us to get substantial coverage of many $\mathrm{C}++$ code-bases using COM.

\section{B. Instrumentations}

The instrumentation mechanism in HAVOC was extended to support some object-oriented constructs. These include (a) instrumenting all instance methods declared in a given class, and (b) instrumenting an instance method in all classes.

The first feature is useful for annotating class invariants, by instrumenting all instance methods in a class with a precondition and postcondition. In addition, the user can remove the constructors and destructors from the set by using a set of patterns that are excluded. For example,

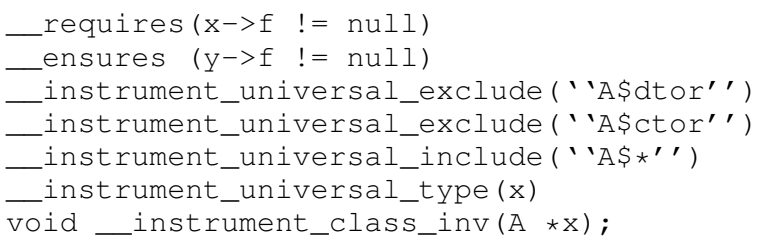

instruments all methods in the class A (denoted by the -include pattern) except the constructor and destructor (denoted by the _exclude patterns) require and ensure that a field $f$ is non-null for the receiver ("this") object. Instance methods that take additional objects of type A or static methods that take an object of type $A$ have to be manually excluded though.

The second feature is often useful for getting the effect of annotation inheritance, where annotation on a virtual method is inherited by the overriding methods of all derived classes. All instance methods with the name foo can be specified with the pattern ' ' $\star \$ f \circ o '$ '. However, we currently do not provide any support for checking that derived classes only weaken preconditions and strengthen postconditions - it is left to the user to enforce.

\section{Inference}

HAVOC used the Houdini algorithm [4] to choose inductive invariants from a set of candidates. Although the inference was successful in scaling to modules with several hundred to a few thousand procedures [2], it did not scale to the modules that had several hundred thousand procedures measuring several million lines of code. In this section, we discuss the improvements made to make the inference scalable to these modules.

1) Persisting fewer Boogie files: The approach in HAVOC generated a single BoogiePL file on disk containing the definition of all the procedures, and then invoked the Houdini procedure inside Boogie. However, creating a Boogie file with almost a million procedure did not scale as HAVOC crashed due to memory blowup during the generation of the Boogie file, and Boogie could not load such a file and perform VC generation. Instead, we first changed the flow to generate a Boogie file per procedure, and fed Boogie a list of Boogie files. This avoided the memory blowup in HAVOC, but still caused Boogie to take a long time.
Our first observation is that for the purpose of Houdini inference, one can safely filter procedures that do not have any (a) candidate assertions inside the body of the procedure, (b) does not have any candidate postconditions, (c) does not have (immediate) callees that have a candidate precondition. This is because analyzing such procedure will always return "verified" as there are no assertions to check. This simple optimization allowed us to reduce the number of Boogie files used during the inference by at least 2 orders of magnitude, when the annotations were sparse.

2) Two-level Houdini algorithm: In spite of the dramatic reduction in the number of procedures being analyzed during Houdini, we were still left with several tens of thousand of procedures to analyze, and maintaining all the procedures (and their VCs) in memory during Houdini exceeded memory. To alleviate it, we designed a two-level Houdini algorithm that uses a cache to only pass a small set of procedures to the Houdini procedure.

Given a set of procedures $P$ with candidate annotations $C$ and a cache size $n$, the algorithm operates as follows.

1) It first initializes a work list $W$ with all the procedures in $P$.

2) At each stage, it removes a set $S$ of $n$ procedures from $W$ (or all procedures if $|W| \leq n$ ) and invokes Houdini on $S$.

3) Houdini removes a (possibly empty) subset of annotations $R \subseteq C$ after analyzing $S$.

4) For each removed candidate $c \in R$, we update the worklist as follows: (a) if $c$ is a precondition of a method $p \notin S$, then we add $p$ to $W$, and (b) if $c$ is a postcondition of a method $p$ then we add any of its caller $q$ that is not present in $S$.

5) At the same time, $C$ is updated to $C \backslash R$ by removing the candidates removed by Houdini.

6) The method is repeated until $W$ is empty.

It is not difficult to show that when $W$ is empty, then the set of candidates in $C$ can be proved modularly.

The size of the cache influences the overall runtime as the larger the cache, the more chance the (inner) Houdini algorithm gets to perform optimizations, and thus the outer loop converges faster. On the other hand, making the cache large increases the memory consumption for Houdini. We have observed that the memory requirement is really a function of the size of the procedures instead of the number of procedures. For a given problem where the size of the largest procedure is $k$, it is useful to set $n$ so that $n * k$ does not exceed the memory alloted to the process. For most of our experiments, $n$ is set between 20 and 100 .

3) Candidates on roots and leaves: It is well-known that if the set of candidate preconditions on root procedures (that have no callees) or the set of candidate postconditions on leaf procedures (that have no body) are inconsistent, then Houdini can infer annotations that may not hold. Previously, the users of HAVOC manually ensured that the root procedures have no candidate preconditions and leaf procedures have no candidate postconditions. This was an expensive process as determining 
the roots can be tricky when certain procedures are targets of function pointers. To simplify matters, HAVOC-LITE first removes candidate preconditions from the root and candidate postconditions from the leaves of the call graph presented to it for annotation inference. This substantially improves the usability of the inference for the user.

\section{Other}

In addition to the above enhancements, a number of other usability issues were addressed. First, we added a mechanism to insert annotations completely non-intrusively — i.e. the source tree did not have to be modified. Annotations were first compiled with type and procedure declarations and then linked with the definitions later. This greatly improved the adoption in teams that did not want to modify the source code, even to include an annotation header file. The separate compilation of the annotation file also allowed us to correct annotation parse errors quicker. HAVOC-LITE also added bitvector support by interpreting the scalars and pointers as fixedsize bit-vectors, and using the theory of bit-vectors inside SMT solver Z3. Earlier implementation in HAVOC used unbounded integers and could not model the bitwise operations accurately. Finally, the user can associate custom strings and identifiers for warning messages that are displayed on assertion failures - this allowed easier triaging of warnings when multiple properties were checked in a single run.

\section{Evaluation}

In this section, we go through the evaluation of the capabilities of HAVOC-LITE to detect variations of known software problems that are commonly reported in Microsoft products. Security holes belonging to these vulnerability classes, when reported by independent security researchers, give rise to security bulletins and general distribution release of fixes. Such bulletins are very costly for software vendors and users, in particular for businesses with hundreds or thousands of computers, whose activity is interrupted while the update is performed. As such, each bulletin must ensure that it covers not only the originally reported security problem, but variations of the problem that may be more easily identified by third party researchers after the bulletin is published. Extended static checking helps software vendors increase coverage of such reactive analysis.

\section{A. Checked properties}

We have evaluated the tool on the large scale on multiple heterogeneous properties across multiple products written in $\mathrm{C}$ and $\mathrm{C}++$, summarized in Figure 8 . The properties were derived by studying a set of security vulnerabilities reported in a set of recent MSRC bulletins ( [13], [14], [15], [16], [17], [18], [19], [20]). We avoid relating the properties to specific MSRC cases to avoid disclosing any confidential information not available in the bulletins.

Some of the properties we describe in this table are in fact a family of properties. For example, zero-sized allocations is an umbrella denomination for calls to dynamic memory allocation APIs such as the user-mode malloc, the kernel-mode ExAllocatePool, dynamic array allocations via the new operator, or object constructors taking an integer parameter that is used to allocate an internal buffer used within this class object.

Other interesting properties come from expected contracts of special VARIANT object (recall the example from Section II). The type names have been changed to avoid unintended consequences of releasing such information. Such data structures holding run-time type information can be the source of multiple security vulnerabilities if they are initialized or used incorrectly.

Another interesting property ("Interface reference counting") arises from the need to enforce the usage of interfaces in object-oriented programs. An interface can be seen as a structure that holds a fixed list of function pointers. Interfaces are usually reference counted and released once their expected life time has been reached. Our introductory example in the overview section was taken from this class of security vulnerabilities affecting object-oriented software.

The property of library path qualification captures the intent that no binary dependence should be loaded from an unknown location under the threat of loading untrusted code (potentially from a remote location if the path is in the UNC format such as "\\remote $\backslash$ machine $\backslash$ untrusted.dll"). On the other hand, a preceding call to a trusted path-retrieving API such as GetSystemDirectory acts as a sanitizer, as it provides a proof that such path is prefixed by a string of the form "c : \windows \system $32 "$.

\section{B. Results}

The result of checking those properties over the course of a year is presented in Figure 9. We identified around 70 vulnerabilities in critical software components using a build server equipped with 48 cores and 96GB of RAM to perform this analysis. While the big number of cores significantly speeds up the intra-procedural analysis, the inter-procedural does not currently benefit from it due to the sequential implementation. The "Check" time is the time to only check the annotations intra-procedurally, including inferred annotations if any. The "Inference" time is the time taken to perform the inter-procedural annotation inference. We also report a few other vulnerabilities for other properties later in this section - we did not perform a thorough evaluation of times etc. for these properties.

Each analyzed property corresponds to a class of security vulnerability previously discovered and fixed in the analyzed code base via general distribution release of security bulletins. We only applied a particular property checking to the code bases that were affected by the property - this explains the difference in code size for each experiment. While some of the properties (e.g. Library path qualification) affected all usermode code bases, others (such as the DOM property) only affects the core browser engine. Other generic COM properties such as the VARIANT initialization and type safety checking 


\begin{tabular}{|l|l|}
\hline Properties & Description \\
\hline Zero-sized allocations & Dynamic memory allocations should never be of size 0. \\
\hline Empty array construction & There is always at least one element in new[] allocated arrays. \\
\hline VARIANT initialization & VARIANT structures should never be used without initialization. \\
\hline VARIANT type safety & VARIANT union fields should never be referenced without proper field type value. \\
\hline Interface reference counting & Interfaces should never be released without prior reference or initialization. \\
\hline Library path validation & Dependencies modules should never be loaded without fully qualified path. \\
\hline DOM information disclosure & DOM accessors only returns success on successful completion. \\
\hline
\end{tabular}

Fig. 8. Checked security properties

\begin{tabular}{|l|l|l|l|l|l|}
\hline Properties & LOC & Procnum & Bugs & $\begin{array}{l}\text { Check } \\
\text { time }\end{array}$ & $\begin{array}{l}\text { Inference } \\
\text { time }\end{array}$ \\
\hline Zero-sized allocations & $2.8 \mathrm{M}$ & $58 \mathrm{~K}$ & 9 & $3 \mathrm{~h} 14$ & $3 \mathrm{~h} 22$ \\
\hline Empty array constructor & $1.2 \mathrm{M}$ & $3.1 \mathrm{~K}$ & 0 & $26 \mathrm{~m}$ & $6 \mathrm{~m} 13$ \\
\hline VARIANT initialization & $6.5 \mathrm{M}$ & $196 \mathrm{~K}$ & 5 & $5 \mathrm{~h} 03$ & $11 \mathrm{~h} 40$ \\
\hline VARIANT type safety & $6.5 \mathrm{M}$ & $196 \mathrm{~K}$ & 8 & $5 \mathrm{~h} 03$ & $11 \mathrm{~h} 40$ \\
\hline Interface reference counting & $2 \mathrm{M}$ & $11.2 \mathrm{~K}$ & 4 & $2 \mathrm{~h} 26$ & $20 \mathrm{~h}$ \\
\hline Library path qualification & $20 \mathrm{M}$ & Millions & 35 & $5 \mathrm{~d}$ & N/A \\
\hline DOM information disclosure & $2.5 \mathrm{M}$ & Hundreds & 2 & $1 \mathrm{~h} 42$ & N/A \\
\hline
\end{tabular}

Fig. 9. Summary of results.

and the interface reference counting were ran on a set of large user-mode code bases making heavy use of such features. Two properties affecting the VARIANT structures were checked together on the same code-base, hence the check time and inference time are the same for both.

Note that inference information is not available for two properties: the library path qualification acted on tens of millions of lines of code, a size for which inference is not able to scale due to the sequential implementation. Likewise, the DOM information disclosure property only affected class accessors of the form CBrowserObj::get_attr totalizing only a few hundred methods to check in just 8 different classes for which the manual warning review process was fast enough without inference.

In addition to the properties described in Figure 8, we also uncovered security critical bugs from other checks. We describe a couple of them next.

1) We also applied HAVOC (earlier version) to check user-kernel pointer probing on the Windows application APIs - to ensure that user-mode pointer should always be validated by specific Probe* APIs before being dereferenced in the kernel [20]. The effort on around $300 \mathrm{KLOC}$ revealed another 7 bugs that were fixed. These bugs are counted towards the 70 bugs that we report.

2) We also discovered a set of errors during the translation from $\mathrm{C}$ source files to the well-typed BoogiePL language. One of the checked kernel driver was using the following mechanism at multiple locations:

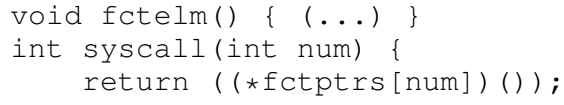

where syscall is a kernel entry-point returning an integer error code to the unprivileged user, and $f_{c t e} \mathrm{~lm}$ is a void-returning function, which is part of a function pointer array fctptrs whose elements were supposed to return an integer. Such unsafe function pointer elements in the array were possible at compilation time due to the use of unsafe function pointer casts. Such defect can result in information disclosure security vulnerabilities since the return value (on the Pentium architecture, held in the eax register) is uninitialized in the $\mathrm{fctelm}$ function and used for another purpose (for example, holding the pointer to sensitive kernel data structures). We discovered around $30+$ bugs in the driver that were fixed. These are in addition to the 70 bugs that were found as variants of existing vulnerabilities.

\section{Inter-procedural annotation inference}

Inference is a useful technique to improve the checker's result when properties depend on the caller function context and the results of callee functions.

Figure 10 shows the difference in number of false positives when the user-guided annotation inference was used. Interprocedural analysis brings improvements in precision and lessen the amount of false positives, but the impact varies by the property. We found that for most sparse properties (such as the API related properties where only small numbers of checks are performed compared to the program size), the number of warnings diminishes by $10 \%$ to $45 \%$ depending on the checked property. A simple example of inference used to check the zero allocation property used generated candidate contracts of the form:

cand_requires (param $!=0)$
cand_ensures (_return $!=0)$ 


\begin{tabular}{|l|l|l|l|l|l|}
\hline Properties & $\begin{array}{l}\text { Warnings } \\
\text { Warnings }\end{array}$ & $\begin{array}{l}\text { Warnings } \\
\text { with inference }\end{array}$ & Improvement & Candidates & $\begin{array}{l}\text { Inferred } \\
\text { candidates }\end{array}$ \\
\hline Zero-sized allocations & 71 & 50 & $29 \%$ & 75162 & 42160 \\
\hline Empty array constructor & 45 & 35 & $22 \%$ & 4024 & 446 \\
\hline VARIANT initialization & 216 & 117 & $45 \%$ & 100924 & 770 \\
\hline VARIANT type safety & 83 & 68 & $18 \%$ & 100924 & 770 \\
\hline Interface reference counting & 746 & $672(3)$ & $10 \%$ & $234 \mathrm{~K}$ & 1671 \\
\hline DOM information disclosure & 82 & N/A & N/A & N/A & N/A \\
\hline Library path qualification & 280 & N/A & N/A & N/A & N/A \\
\hline
\end{tabular}

Fig. 10. Results of running annotation inference. "Candidates" denotes the number of candidate annotations and "Inferred candidates" is the number of annotations that were inferred to hold.

int fet(int param) $\{\ldots\}$

for every function accepting integer parameters and integer return values. Therefore, enforced preconditions of the form:

-requires (size $!=0$ )

void* ExAllocatePool(unsigned int size);

can be checked with knowledge of the inferred (persisted) constraints at function boundaries. In this case, the burden of writing the candidate annotations before the checking phase is performed is very small and provides a good payoff in diminishing the number of false positives. We have applied this methodology to multiple properties as indicated in Figure 10.

Properties such as "interface reference counting" are ones for which inference is the most useful as the number of pointers to be tracked within the target modules is generally quite large. Such pointers can also sometimes passed between modules, which limit the ability of inference to filter out false positives. This is reflected in the false positive ratio of Figure 10. In general, our experiments support the fact that while inference does not suffice to reach a perfect analysis result, its use allow diminishing the burden of warning reviews in an appreciable way for security auditors.

Annotation inference works best when the set of intermediate annotations can be concisely expressed using candidate annotations that can be added with simple instrumentations. A user starts with the property under check, inspects the warnings from the checking and then devises a small set of candidates. Annotation inference is performed and the new set of warnings are noted and new candidates are added. The process remains cost-effective up to a couple of iterations, beyond which the auditor preferred to manually inspect the false alarms.

Cases where inference fails to diminish the number of false positives include functions with aliasing between pointer parameters for which no annotation can be synthesized without introducing more complex conditional constraints. In other cases, the annotations need to talk about type-states of deeply nested fields of parameters, or global variables. While such conditions can be encoded with modest manual effort for individual functions, such a scheme will likely generate too many candidate annotations for the modules under consideration.

\section{Use of unsound modifies clauses}

In addition to using inference, we also performed an evaluation with the use of modifies clauses, where extra assumptions were added telling that the state of the heap did not change when function calls are performed. This is an unsound assumption but has the advantage of bringing down the number of false positives drastically. For example, when checking the interface reference counting property, enabling this option brought the warning number down from 672 (after using inter-procedural inference) to 3 , of which all were valid vulnerabilities. We employed this unsound mechanism for this particular property for two reasons: (1) the set of alarms even after inference was very large, and (2) a random sampling of the warnings revealed that state updates of the interface pointers in callees was responsible for the false alarms. No extra annotations were explicitly necessary for such assumptions, as HAVOC-LITE provides an option through the configuration file. A similar decrease in warning numbers is witnessed on other properties though this option is only deemed necessary when the initial set of (false positive) alerts is large enough to justify losing soundness.

\section{E. Cost-effectiveness and warnings review}

The cost-effectiveness of using an extended static checker varies depending on each property. We found that security properties related to API calls are generally sparse; only a few calls are instrumented with assertions or candidates compared to the total number of lines of code and procedures of the analyzed modules. Checking field dereference also came with a reasonable return on investment as long as the number of fields that were checked for dereferences remained small enough. We found that the number of false positives for sparse properties (such as library path qualification, zero-sized allocations, VARIANT initialization or VARIANT type-safety) was acceptable as we consistently found new vulnerabilities in the reported warning list. Other denser properties (such as the previous attempt of user kernel pointer probing) were harder to check with only $5-10 \%$ of real vulnerabilities among the total list of warnings. We explain this by the need to have every single pointer dereference be instrumented, representing tens of thousands of dereferences on medium sized modules.

Another interesting metric to measure the success of such tool comes from the engineer feedback. We found that, on 
average, a security expert can review between 25 and 50 warnings per day. For some properties involving very deep inference and for which a function containing an alert has a large number of callees, we found that it can take one to four hours to review a single warning. This can also happen in case of very complex control flow that arises within or between multiple components. Such cases often involve indirect call sites via function pointers or virtual methods, including callbacks that sometimes cross a domain boundary (i.e. user-mode call-backs). For such instances of warnings, the help of a debugger is often necessary to understand whether the discovered vulnerable context is feasible, and more importantly, can be controlled by the user. A bug may be benign from the security perspective, if the variable values that can trigger the unsafe behavior are not under the control of the user (for example, the variables can be under control of a trusted third party components on which the analysis was not performed).

Overall, we found that the use of HAVOC-LITE was costeffective compared to pure manual code review. A similar analysis done purely in a manual fashion would have required weeks if not months of work. The ability of static analysis to focus on crucial control locations and specific data manipulations was fundamental in this exercise. The ability to perform inter-procedural inference brought down the warning number consistently, saving hours of warning review to the analyst.

\section{F. Found vulnerabilities}

We now give a few examples of vulnerabilities that we were able to identify using extended static checking, starting with the interface reference counting vulnerability class.

1) Interface reference counting: The first example (Figure 11) of found and fixed vulnerability relates to the reference counting property of interfaces. The COM model makes heavy use of interface pointers, in particular in objectoriented projects like the browser where deep levels of inheritance are used and objects in different hierarchies share some features. Those features are therefore implemented in interfaces that classes can inherit without having to derive from another class. The IUnknown interface serves as a base interface for all other interface and class types (such as CBrowserElement in this example) by implementing three core methods called QueryInterface, AddRef and Release. A call to QueryInterface accepts an interface identifier and returns an array of function pointers that represents the implementation of the desired interface. If no such interface is available in the base object, QueryInterface will return an error. Otherwise, it will return status code S_OK. Note that QueryInterface also performs a call to AddRef on the base class if the query is successful, so that the class is not freed while the program still holds a reference onto one of its interface. A critical safety property of such model states that any pointer on a COM interface written to via a call to QueryInterface should be released by a call to ReleaseInterface after usage, except when the call to QueryInterface failed (in that case, there is nothing to release). The ReleaseInterface API is simply a wrapper to the Release method that adds a wrapping check that ensures that the interface pointer is not NULL (in that case, the function is simply a NO OP).

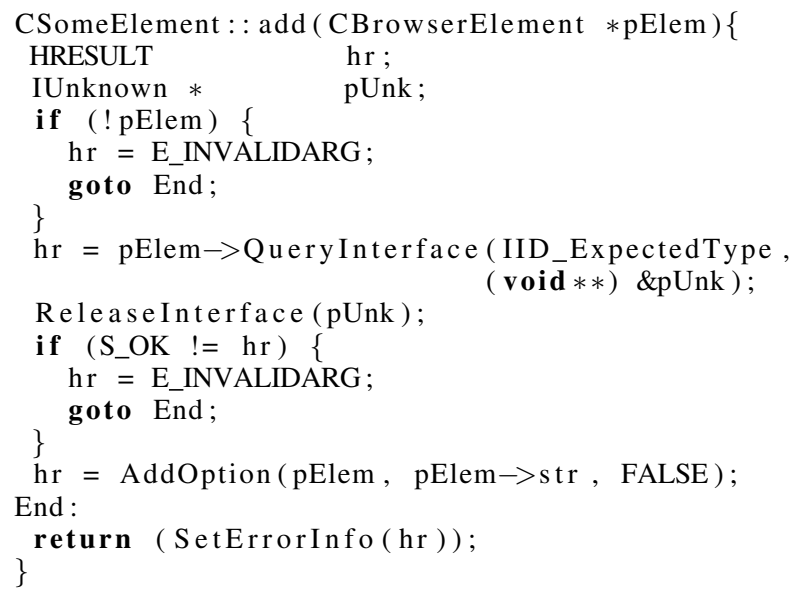

Fig. 11. A real interface reference counting vulnerability (obfuscated)

In this example, this mechanism is used to guarantee that a given browser element $\mathrm{pElem}$ is of the intended type. However, the interface is never used as the looked up content is automatically discarded via the call to ReleaseInterface and the element is simply passed to method AddOption for storage. A vulnerability exists when the QueryInterface method fails and ReleaseInterface is still being called. This is due to (1) the pUnk pointer being uninitialized and (2) the return check being placed after the call to ReleaseInterface. The combination of those two bad coding practices leads to a security vulnerability due to calling method Release on an initialized interface pointer that will later trigger in the form of a use-after-free vulnerabilities. In order to exploit this flaw for untrusted code execution, an attacker would need to control the content of the stack and make the stack offset used by local variable pUnk coincides with the stack offset used by another referencecounted class in a previous stack context (for example, in a function previously called and already returned from, that held a local variable at the same stack offset just before the call to CSomeElement: :add was performed). Such security exploits have already been demonstrated by industry researchers.

2) VARIANT type confusion: The second vulnerability class (Figure 12) that we present in this article is related to the VARIANT data structure. The VARIANT data structure is used in COM applications to transfer data items across generic interfaces. A generic container for arrays of VARIANT structures used in such COM programs is the DISPPARAMS structure. This container type is used, among others, by the IDispatch: : Invoke interface method. A vulnerable specialization of this method is shown below. The CBrowserOp class derives from IDispatch and overloads its Invoke method. This derived method then assumes that the first 


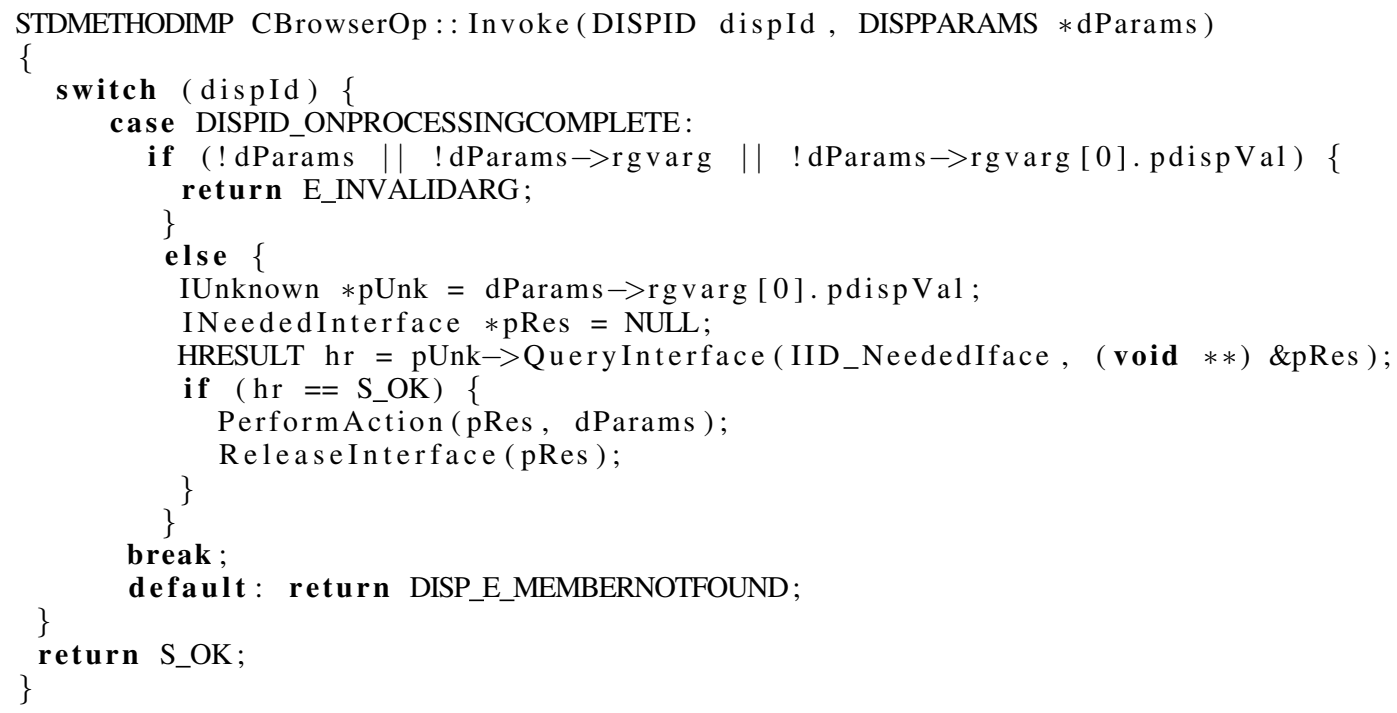

Fig. 12. A real VARIANT type confusion vulnerability (obfuscated)

VARIANT array element is an IUnknown interface pointer (which is the base interface for all COM classes and COM interfaces) and looks up the desired interface (using a call to QueryInterface) needed to perform the effective COM operation. The rest of the VARIANT array elements is then passed to this newly looked-up interface. On success, the action is performed and the interface is properly released.

The safety of VARIANT manipulation relies on testing its $v t$ field to make sure that the contained pointer corresponds to a data type that the interface is expecting and able to treat. Failure to perform such check can be devastating for the security of code, especially if the input parameters are user-controlled. This method fails to perform such check before calling QueryInterface on the IUnknown pointer pUnk. If the first VARIANT structure field dParams $\rightarrow$ rgvarg [0] . pdispVal were to contain another type of interface, a different method than QueryInterface would be called. An attacker could redirect execution onto an instruction pointer of its choice, leading to a likely exploitable memory corruption in the program. The appropriate fix for this vulnerability is to extend the conditional predicate to insert a test $\mathrm{vt}==\mathrm{VT}$ _UNKNOWN | $\mid$ vt $==$ VT_DISPATCH to make sure that the VARIANT structure holds the appropriate interface pointer into which the QueryInterface method is implemented, and returns E_INVALIDARG if this is not the case.

\section{RELATED WORK}

Extended static checking was pioneered for Java by the work of ESC/Java [1] as a means to use program verification methods to provide high coverage of user defined assertions. It has since then been applied to other languages such as Spec\# for C\# [21], and HAVOC for C [2]. Unlike these tools, HAVOC-LITE provides a rich set of instrumentation and inference capabilities to reduce the manual burden for large modules. HAVOC-LITE provides support for most common $\mathrm{C}++$ features used in legacy applications.

Software model checkers such as SLAM [22] offer automatic annotation inference based on predicate abstraction for sparse type-state properties. However, these approaches are not known to scale to modules greater than 100KLOC, thus cannot be applied to most of the modules in this work. Since these tools strive for complete automation, their architecture does not provide mechanisms for users to configure the analysis by providing the set of candidates as HAVOC-LITE provides. Saturn [23] provides precise intra-procedural analysis using SAT solvers, and uses procedure summaries over a set of fixed vocabulary. The tool developer provides appropriate summaries for various properties such as memory leaks, and is not easily configurable. Saturn has been used to check specific security properties such as validation of user-land pointers passed to the kernel [24]; however, this required the careful configuration of the Saturn checker that cannot be expected of an average security expert. Chen et al. [25] apply the model checker MOPS to check similar type-state properties on a million lines of code. However, the properties are restricted to finite state machines and its harder for the users to describe type-state properties on fields of objects.

Hackett et al. [26] provide a checker for buffer overruns in legacy applications using a combination of buffer length annotation and a set of custom rules to check these annotations. The checker uses a custom constraint solver (not modern SMT solvers) using a few simple rules. However, they provide useful heuristics to infer buffer annotations (relating buffers to their lengths) that significantly reduces the annotation effort. However, the technique cannot be readily extended to the properties we discuss in this work and therefore not a good match for security auditors. 
Theorem provers have recently been quite successful in dynamic test generation tools such as DART [27], EXE [28] and SAGE [29]. These techniques leverage existing tests to create path constraints that can be negated to obtain tests for alternate paths. These techniques have revealed several bugs in large applications (in integration testing) or small libraries (in unit testing). However, these approaches are oblivious of the property being checked and aim at providing higher path coverage. These techniques do not use procedure summaries (such as those provided by our candidate annotations) and therefore cannot provide coverage guarantees on the entire attack surface. Since these techniques are primarily based on testing, they suffer from few false alarms. On the other hand, they require setting up complex harnesses and have difficulty being exhaustive for deep components. Besides, several of the security properties (e.g. double free) do not lead to crashes and are consequently harder to detect by testing based tools. Recent work on Automated Exploit Generation [30] attempts both vulnerability checking and input crafting in order to find a code defect and force execution to be redirected on malicious code. Such an approach has been focused on basic security properties such as buffer overflow induced by insecure APIs like strcpy. Such combination of SMT solvers and dynamic test generation is an interesting approach guaranteeing that identified vulnerabilities are real and does not require warning triage. Improvements in performance and expressiveness (such as extending preconditioned symbolic execution [31]) will allow more properties to be checked. However, such framework provides very limited configurability for a security auditor and does not guarantee as much coverage as an extended static checker.

Security properties such as the VARIANT type consistency [32] and the reference counting invariants [33] have previously been studied for COM programs and web browsers via run-time monitoring and unit testing. However, no systematic program analysis was performed to the extent of this work. As such, no guarantee of coverage could be made based upon concrete executions of the program under scrutiny. HAVOCLITE provides security auditors with extended security audit abilities and allow focusing on code locations where such security properties could not be proved. As such, extended static checking provides a much stronger guarantee that no instance of such vulnerability has been left behind.

\section{CONCLUSIONS}

Extended static checking is a good complement to fuzz testing and other data-flow based static analysis techniques. In particular, the inter-procedural inference is a key component in diminishing the number of false positives (sometimes up to $45 \%$ ) to an acceptable level for security experts to review. Nonetheless, the cost associated to running the inference is still high. Our goal is to extend the two-level Houdini algorithm to a distributed version that can invoke multiple versions of the inner Houdini algorithm in parallel. HAVOC-LITE currently does not weigh warning confidence and users have to go through the entire list of alerts to have a faithful understanding of the results - performing warning prioritization is an important next step for HAVOC-LITE.

\section{ACKNOWLEDGMENTS}

We would like to thank the anonymous reviewers and our shepherd Patrick McDaniel for improving the presentation of the paper. We are grateful to Mark Wodrich and Ali Rahbar from the MSRC team for their help in deploying the tool, and to Shaz Qadeer for his help in porting the tool to the latest build environment in Windows.

\section{REFERENCES}

[1] C. Flanagan, K. R. M. Leino, M. Lillibridge, G. Nelson, J. B. Saxe, and R. Stata, "Extended static checking for Java," in Programming Language Design and Implementation (PLDI'O2), 2002, pp. 234-245.

[2] T. Ball, B. Hackett, S. K. Lahiri, S. Qadeer, and J. Vanegue, "Towards scalable modular checking of user-defined properties," in Verified Software: Theories, Tools, Experiments (VSTTE '10), vol. LNCS 6217, 2010, pp. $1-24$.

[3] Satisfiability Modulo Theories Library (SMT-LIB), "Available at http://goedel.cs.uiowa.edu/smtlib/."

[4] C. Flanagan and K. R. M. Leino, "Houdini, an annotation assistant for ESC/Java," in International Symposium of Formal Methods Europe (FME '01), 2001, pp. 500-517.

[5] M. Dahlweid, M. Moskal, T. Santen, S. Tobies, and W. Schulte, "Vcc: Contract-based modular verification of concurrent c," in International Conference on Software Engineering, (ICSE '09), Companion Volume, 2009, pp. 429-430.

[6] B. Chess, "Improving computer security using extended static checking," in IEEE Symposium on Security and Privacy, 2002, pp. 160-

[7] Component Object Model (COM), "Available at http://www.microsoft.com/com/default.mspx."

[8] C. Flanagan, K. R. M. Leino, M. Lillibridge, G. Nelson, J. B. Saxe, and R. Stata, "Extended static checking for Java," in Programming Language Design and Implementation (PLDI'02), 2002, pp. 234-245.

[9] R. DeLine and K. R. M. Leino, "BoogiePL: A typed procedural language for checking object-oriented programs," Microsoft Research, Tech. Rep. MSR-TR-2005-70, 2005.

[10] J. Condit, B. Hackett, S. K. Lahiri, and S. Qadeer, "Unifying type checking and property checking for low-level code," in Principles of Programming Languages (POPL '09), 2009, pp. 302-314.

[11] M. Barnett and K. R. M. Leino, "Weakest-precondition of unstructured programs." in Program Analysis For Software Tools and Engineering (PASTE '05), 2005, pp. 82-87.

[12] S. K. Lahiri, S. Qadeer, and Z. Rakamaric, "Static and precise detection of concurrency errors in systems code using smt solvers," in Computer Aided Verification (CAV '09), vol. LNCS 5643, 2009, pp. 509-524.

[13] MSRC MS10-054, "Microsoft security bulletin ms10-054 critical," 2010. [Online]. Available: http://technet.microsoft.com/enus/security/bulletin/MS10-054

[14] MSRC MS11-092, "Microsoft security bulletin ms11-092 critical," 2011. [Online]. Available: http://technet.microsoft.com/enus/security/bulletin/MS11-092

[15] MSRC MS09-034, "Microsoft security bulletin ms09-034 critical," 2009. [Online]. Available: http://technet.microsoft.com/enus/security/bulletin/MS09-034

[16] MSRC MS11-093, "Microsoft security bulletin ms11-093 important," 2011. [Online]. Available: http://technet.microsoft.com/enus/security/bulletin/MS11-093

[17] MSRC MS11-018, "Microsoft security bulletin ms11-018 critical," 2011. [Online]. Available: http://technet.microsoft.com/enus/security/bulletin/MS11-018

[18] MSRC MS12-074, "Microsoft security bulletin ms12-074 critical," 2012. [Online]. Available: http://technet.microsoft.com/enus/security/bulletin/MS12-074

[19] MSRC MS11-050, "Microsoft security bulletin ms11-050 critical," 2011. [Online]. Available: http://technet.microsoft.com/enus/security/bulletin/MS11-050

[20] MSRC MS08-066, "Microsoft security bulletin ms08-066 important," 2008. [Online]. Available: http://technet.microsoft.com/enus/security/bulletin/MS08-066 
[21] M. Barnett, K. R. M. Leino, and W. Schulte, "The Spec\# programming system: An overview." in Construction and Analysis of Safe, Secure and Interoperable Smart Devices (CASSIS '05), 2005, pp. 49-69.

[22] T. Ball, R. Majumdar, T. Millstein, and S. K. Rajamani, "Automatic predicate abstraction of C programs," in Programming Language Design and Implementation (PLDI '01), 2001, pp. 203-213.

[23] A. Aiken, S. Bugrara, I. Dillig, T. Dillig, B. Hackett, and P. Hawkins, "An overview of the Saturn project," in Workshop on Program Analysis for Software Tools and Engineering (PASTE '07), 2007, pp. 43-48.

[24] S. Bugrara and A. Aiken, "Verifying the safety of user pointer dereferences," in IEEE Symposium on Security and Privacy, 2008, pp. 325-338.

[25] H. Chen, D. Dean, and D. Wagner, "Model checking one million lines of c code," in Proceedings of the Network and Distributed System Security Symposium (NDSS'04), 2004.

[26] B. Hackett, M. Das, D. Wang, and Z. Yang, "Modular checking for buffer overflows in the large," in International Conference on Software Engineering (ICSE '06), 2006, pp. 232-241.

[27] P. Godefroid, N. Klarlund, and K. Sen, "DART: Directed automated random testing." in Programming Language Design and Implementation (PLDI '05). ACM, 2005, pp. 213-223.

[28] C. Cadar, V. Ganesh, P. M. Pawlowski, D. L. Dill, and D. R. Engler, "Exe: automatically generating inputs of death," in ACM Conference on Computer and Communications Security, 2006, pp. 322-335.

[29] P. Godefroid, M. Y. Levin, and D. A. Molnar, "Sage: whitebox fuzzing for security testing," Commun. ACM, vol. 55, no. 3, pp. 40-44, 2012.

[30] H. B. L. T. Avgerinos Thanassis, Cha Sang Kil and B. David, "Aeg: Automatic exploit generation," ser. Network and Distributed System Security Symposium, 2011.

[31] S. K. Cha, T. Avgerinos, A. Rebert, and D. Brumley, "Unleashing mayhem on binary code," in Proceedings of the 2012 IEEE Symposium on Security and Privacy, ser. SP '12. Washington, DC, USA IEEE Computer Society, 2012, pp. 380-394. [Online]. Available: http://dx.doi.org/10.1109/SP.2012.31

[32] M. Dowd, R. Smith, and D. Dewey, "Attacking interoperability," ser. Blackhat USA briefings, 2009.

[33] S. Chen, H. Chen, and M. Caballero, "Residue objects: a challenge to web browser security," in Proceedings of the 5th European conference on Computer systems, ser. EuroSys '10. ACM, 2010, pp. 279-292. 